\title{
Chapter 16 \\ Inducing Enantiosensitive Permanent Multipoles in Isotropic Samples with Two-Color Fields
}

\author{
Andres F. Ordonez and Olga Smirnova
}

\begin{abstract}
We find that two-color fields can induce field-free permanent dipoles in initially isotropic samples of chiral molecules via resonant electronic excitation in a one- $3 \omega$-photon versus three- $\omega$-photons scheme. These permanent dipoles are enantiosensitive and can be controlled via the relative phase between the two colors. When the two colors are linearly polarized perpendicular to each other, the interference between the two pathways induces excitation sensitive to the molecular handedness and orientation, leading to uniaxial orientation of the excited molecules and to an enantio-sensitive permanent dipole perpendicular to the polarization plane. We also find that although a corresponding one- $2 \omega$-photon versus two- $\omega$-photons scheme cannot produce enantiosensitive permanent dipoles, it can produce enantiosensitive permanent quadrupoles that are also controllable through the two-color relative phase.
\end{abstract}

\section{Introduction}

Chirality (handedness) is the geometrical property that allows us to distinguish a left hand from a right hand. Like hands, many molecules have two possible versions which are non-superimposable mirror images of each other (opposite enantiomers). This "extra degree of freedom" stemming from the reduced symmetry (lack of improper symmetry axes) of chiral molecules leads to interesting behavior absent in achiral molecules [1-6] with profound implications for biology [7, 8]. Furthermore, since

\footnotetext{
A. F. Ordonez

Max-Born-Institut, Max-Born-Str. 2A, 12489 Berlin, Germany

e-mail: ordonez@mbi-berlin.de

O. Smirnova $(\varangle)$

Max-Born-Institut, Max-Born-Str. 2A, 12489 Berlin, Germany

Technische Universität Berlin, Straße des 17. Juni 135, 10623 Berlin, Germany

e-mail: smirnova@mbi-berlin.de

B. Friedrich and H. Schmidt-Böcking (eds.), Molecular Beams in Physics and Chemistry, https://doi.org/10.1007/978-3-030-63963-1_16
} 


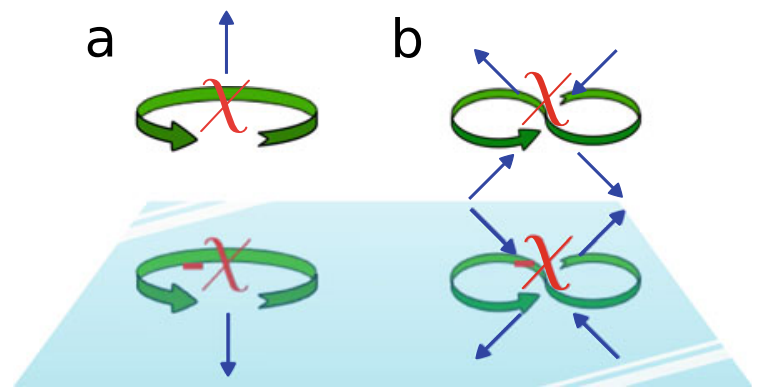

Fig. 1 Symmetry in $\omega$ and $\omega-2 \omega$ setups. a A circularly polarized field (circular arrow) interacts with an isotropic sample of chiral molecules (represented by $\chi$ ) and produces a net photoelectron current (in general a vectorial signal) perpendicular to the polarization plane (arrow pointing up). The mirror reflection shows that the interaction of the same field with the opposite enantiomer (represented by $-\chi$ ) yields the opposite current. b A field with its fundamental and second harmonic linearly polarized perpendicular to each other ( $\infty$-like arrow) interacting with an isotropic chiral sample produces a quadrupolar photoelectron current (in general a quadrupolar signal). The mirror reflection shows that the interaction of the same field with the opposite enantiomer yields the opposite current. In both (a) and (b), the reversal of the signal when the polarization is changed follows from considering a rotation of $180^{\circ}$ (not shown) of the full system, which changes the polarization but not the isotropic sample (see Figs. 2-4 in Ref. [14])

opposite enantiomers share fundamental properties like their mass and their energy spectrum, one must often rely precisely on this chiral behavior to tell opposite enantiomers apart - a task of immense practical importance in chemistry $[9,10]$.

An example of this chiral behavior is the phenomenon known as photoelectron circular dichroism (PECD) [4, 11-13], which consists in the generation of a net photoelectron current from an isotropic sample of chiral molecules irradiated by circularly polarized light [14-16]. This photoelectron current, which results from different amounts of photoelectrons being emitted in opposite directions, is directed along the normal to the polarization plane (because of the overall cylindrical symmetry) and changes sign when either the enantiomer or the circular polarization is reversed (see Fig. 1a). Importantly, PECD occurs within the electric-dipole approximation, which makes typical PECD signals orders of magnitude stronger than traditional enantiosensitive signals, such as circular dichroism (CD), which rely on interactions beyond the electric-dipole approximation $[10,17]$. Furthermore, the electric-dipole approximation also rules out any influence of the wave vector of the incident light and hence of the momentum of the photons.

Given that: the molecules are randomly oriented in space, the electric field is circularly polarized, and the momentum of the photon does not play any role in PECD; it is only natural to wonder why does a net current of photoelectrons perpendicular to the polarization plane occur? From the point of view of symmetry, the question would be instead what symmetry prevents this current from taking place in the case of achiral molecules? The answer is simple: in the electric-dipole approximation ${ }^{1}$ the

\footnotetext{
${ }^{1}$ Beyond the electric-dipole approximation the wave vector of the light breaks reflection symmetry.
} 
system consisting of isotropic achiral molecules together with the circularly polarized electric field is symmetric with respect to reflection in the polarization plane $^{2}$ and therefore the current normal to the polarization plane must vanish. When achiral molecules are replaced by chiral molecules, this mirror symmetry is broken and the PECD current emerges [14].

While this symmetry analysis does not provide an answer in terms of the specific mechanism, the insight it provides applies to several other closely related effects occurring within the electric dipole approximation, which rely on electric field polarizations confined to a plane and yield enantiosensitive vectorial responses perpendicular to that plane $[3,14,18-23]$. For example, if the photon energy of the circularly polarized light is not enough to ionize the molecule, the lack of reflection symmetry due to the chiral molecules leads to oscillating bound currents normal to the polarization plane [14, 23]. In this case, the current results from the excitation of bound states and the associated oscillation of the expected value of the electric dipole operator. The enantiosensitivity is reflected in the phase of the oscillations, which are out of phase in opposite enantiomers.

Analogously, one may also expect that it should be possible to induce permanent electric dipoles (i.e. non-vanishing zero-frequency components of the expected value of the electric dipole operator) normal to the polarization plane and with opposite directions for opposite enantiomers. Indeed, such static electric dipoles have been investigated in the context of optical rectification [24-28], where two excited states close in energy are resonantly excited with monochromatic circularly polarized light. Very recently enantiosensitive static dipoles have also been studied in the context of molecular orientation induced by intense off-resonant light pulses [29-32]. Such light pulses excite rotational dynamics and cause orientation of one of the molecular axes that persists after the pulse is over. Here we show that field-free enantiosensitive permanent electric dipoles and the associated orientation can also be induced in the context of purely electronic excitation on ultrafast time-scales, without relying on rotational dynamics. We achieve this via interference of one- and three-photon excitation pathways.

Quite recently an extension of single-color PECD to two-color $\omega$ - $2 \omega$ fields with orthogonal linear polarizations has been observed [33-35] (see Fig. 1b). As we discuss in Refs. [36, 37], this is an example of how molecular chirality can be reflected not only in scalar (e.g. CD) and vectorial observables (e.g. PECD), but also in higher-rank tensor observables. Here we show that two-color $\omega$ - $2 \omega$ fields with linear polarizations perpendicular to each other can induce enantiosensitive permanent quadrupoles in samples of isotropic chiral molecules.

\footnotetext{
${ }^{2}$ Note that circularly polarized light is not chiral within the electric-dipole approximation, and therefore the chirality of the light itself does not play a role in PECD [14].
} 


\section{Exciting an Enantiosensitive Permanent Dipole}

Consider the excitation scheme depicted in Fig. 2, where the interference of contributions from a one-3 3 -photon pathway and a three- $\omega$-photon pathway control the population of the state $|3\rangle$ of a chiral molecule. For simplicity, we first consider excitation via intermediate resonances in states $|1\rangle$ and $|2\rangle$. The presence of resonances in these states is not essential, as discussed later, but simplifies the analysis. The field is assumed to have the form

$$
\boldsymbol{E}^{\mathrm{L}}(t)=F(t)\left(\boldsymbol{E}_{\omega}^{\mathrm{L}} e^{-i \omega t}+\boldsymbol{E}_{3 \omega}^{\mathrm{L}} e^{-3 i \omega t}\right)+\text { c.c. },
$$

where $F(t)$ is a smooth envelope, $\boldsymbol{E}_{\omega}^{\mathrm{L}}$ and $\boldsymbol{E}_{3 \omega}^{\mathrm{L}}$ specify the polarizations and phases of each frequency, and the $\mathrm{L}$ and $\mathrm{M}$ superscripts indicate vectors and functions in the laboratory frame and in the molecular frame, respectively. For a given molecular orientation $\varrho \equiv \alpha \beta \gamma$, where $\alpha \beta \gamma$ are the Euler angles, the wave function after the interaction is

$$
\Psi^{\mathrm{M}}\left(\boldsymbol{r}^{\mathrm{M}}, \varrho\right)=\sum_{i=0}^{3} a_{i}(\varrho) e^{-i \omega_{i} t} \psi_{i}^{\mathrm{M}}\left(\boldsymbol{r}^{\mathrm{M}}\right),
$$

where $\psi_{i}^{\mathrm{M}}\left(\boldsymbol{r}^{\mathrm{M}}\right)$ is the coordinate representation of state $|i\rangle$ in the molecular frame. In the perturbative regime we have

$$
a_{3}(\varrho)=A_{3}^{(1)}\left[\boldsymbol{d}_{3,0}^{\mathrm{L}}(\varrho) \cdot \boldsymbol{E}_{3 \omega}^{\mathrm{L}}\right]+A_{3}^{(3)}\left[\boldsymbol{d}_{3,2}^{\mathrm{L}}(\varrho) \cdot \boldsymbol{E}_{\omega}^{\mathrm{L}}\right]\left[\boldsymbol{d}_{2,1}^{\mathrm{L}}(\varrho) \cdot \boldsymbol{E}_{\omega}^{\mathrm{L}}\right]\left[\boldsymbol{d}_{1,0}^{\mathrm{L}}(\varrho) \cdot \boldsymbol{E}_{\omega}^{\mathrm{L}}\right],
$$

where $A_{3}^{(1)}$ and $A_{3}^{(3)}$ are first- and third-order coupling constants that depend on the detunings and the envelope (see Appendix). Analogous expressions apply for the other amplitudes $a_{i}$. The transition dipoles $\boldsymbol{d}_{i, j}^{\mathrm{M}} \equiv\left\langle\psi_{i}^{\mathrm{M}}\left(\boldsymbol{r}^{\mathrm{M}}\right)\left|\boldsymbol{d}^{\mathrm{M}}\right| \psi_{j}^{\mathrm{M}}\left(\boldsymbol{r}^{\mathrm{M}}\right)\right\rangle$ are fixed in the molecular frame and have been expressed in the laboratory frame using the rotation matrix $R(\varrho)$ according to $\boldsymbol{d}_{i, j}^{\mathrm{L}}(\varrho)=R(\varrho) \boldsymbol{d}_{i, j}^{\mathrm{M}}$.

Fig. 2 Excitation scheme used to produce an enantiosensitive permanent dipole in an isotropic sample of chiral molecules

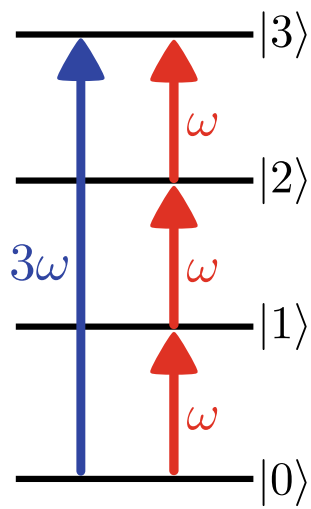


The expected value of the electric dipole operator in the molecular frame $\left\langle\boldsymbol{d}^{\mathrm{M}}(\varrho)\right\rangle$ $\equiv\left\langle\Psi^{\mathrm{M}}\left(\boldsymbol{r}^{\mathrm{M}}, \varrho\right)\left|\boldsymbol{d}^{\mathrm{M}}\right| \Psi^{\mathrm{M}}\left(\boldsymbol{r}^{\mathrm{M}}, \varrho\right)\right\rangle$ has a zero-frequency component of the form

$$
\left\langle\boldsymbol{d}^{\mathrm{M}}(\varrho)\right\rangle_{\omega=0}=\sum_{i=0}^{3}\left|a_{i}(\varrho)\right|^{2} \boldsymbol{d}_{i, i}^{\mathrm{M}},
$$

i.e. the permanent dipole for a given molecular orientation is the sum of the permanent dipoles of each state weighted by their orientation-dependent populations at the end of the pulse.

Transforming $\left\langle\boldsymbol{d}^{\mathrm{M}}(\varrho)\right\rangle_{\omega=0}$ to the laboratory frame and averaging over all molecular orientations yields the permanent dipole

$$
\left\langle\boldsymbol{d}^{\mathrm{L}}\right\rangle_{\omega=0} \equiv \int \mathrm{d} \varrho\left\langle\boldsymbol{d}^{\mathrm{L}}(\varrho)\right\rangle_{\omega=0} .
$$

The contribution of state $|3\rangle$ to this expression reads as ${ }^{3}$

$$
\left\langle\boldsymbol{d}_{3}^{\mathrm{L}}\right\rangle_{\omega=0} \equiv \int \mathrm{d} \varrho\left|a_{3}(\varrho)\right|^{2} \boldsymbol{d}_{3,3}^{\mathrm{L}}(\varrho)=A_{3}^{(1) *} A_{3}^{(3)} \chi_{3} \boldsymbol{Z}^{\mathrm{L}}+\text { c.c. },
$$

where $\int \mathrm{d} \varrho \equiv \int_{0}^{2 \pi} \mathrm{d} \alpha \int_{0}^{\pi} \mathrm{d} \beta \int_{0}^{2 \pi} \mathrm{d} \gamma / 8 \pi^{2}$ is the integral over all molecular orientations and we defined

$$
\begin{gathered}
\chi_{i} \equiv \frac{1}{30}\left[\left(\boldsymbol{d}_{2,1}^{\mathrm{M}} \cdot \boldsymbol{d}_{1,0}^{\mathrm{M}}\right) \boldsymbol{d}_{3,2}^{\mathrm{M}}+\left(\boldsymbol{d}_{3,2}^{\mathrm{M}} \cdot \boldsymbol{d}_{1,0}^{\mathrm{M}}\right) \boldsymbol{d}_{2,1}^{\mathrm{M}}+\left(\boldsymbol{d}_{3,2}^{\mathrm{M}} \cdot \boldsymbol{d}_{2,1}^{\mathrm{M}}\right) \boldsymbol{d}_{1,0}^{\mathrm{M}}\right] \cdot\left(\boldsymbol{d}_{3,0}^{\mathrm{M}} \times \boldsymbol{d}_{i, i}^{\mathrm{M}}\right) \\
\boldsymbol{Z}^{\mathrm{L}} \equiv\left(\boldsymbol{E}_{\omega}^{\mathrm{L}} \cdot \boldsymbol{E}_{\omega}^{\mathrm{L}}\right)\left(\boldsymbol{E}_{\omega}^{\mathrm{L}} \times \boldsymbol{E}_{3 \omega}^{\mathrm{L} *}\right)
\end{gathered}
$$

$\chi_{3}$ is a rotationally invariant molecular pseudoscalar, i.e. a molecular quantity independent of the molecular orientation. It has opposite signs for opposite enantiomers and vanishes for achiral molecules; $\chi_{3}$ encodes the enantiosensitivity of $\left\langle\boldsymbol{d}_{3}^{\mathrm{L}}\right\rangle_{\omega=0}$. Selection rules for $\chi_{3}$ can be directly read off from Eq. (7). In particular, it vanishes if $\boldsymbol{d}_{3,0}^{\mathrm{M}}$ and $\boldsymbol{d}_{3,3}^{\mathrm{M}}$ are collinear. $\boldsymbol{Z}^{\mathrm{L}}$ is a light pseudovector-it is a vector that depends only on the light's polarization and is invariant under the inversion operation; $Z^{\mathrm{L}}$ determines the direction of $\left\langle\boldsymbol{d}_{3}^{\mathrm{L}}\right\rangle_{\omega=0}$. Selection rules for $\boldsymbol{Z}^{\mathrm{L}}$ can be read off directly from Eq. (8). In particular, it vanishes if $\omega$ is circularly polarized $\left(\boldsymbol{E}_{\omega}^{\mathrm{L}} \cdot \boldsymbol{E}_{\omega}^{\mathrm{L}}=0\right)$ or if $\omega$ and $3 \omega$ are linearly polarized parallel to each other $\left(\boldsymbol{E}_{\omega}^{\mathrm{L}} \times \boldsymbol{E}_{3 \omega}^{\mathrm{L} *}=0\right)$.

For example, if we choose $\omega$ and $3 \omega$ linearly polarized perpendicular to each other, say $\boldsymbol{E}_{\omega}^{\mathrm{L}}=\boldsymbol{x}^{\mathrm{L}}$ and $\boldsymbol{E}_{3 \omega}^{\mathrm{L}}=e^{-i \phi} \boldsymbol{y}^{\mathrm{L}}$, with $\boldsymbol{x}^{\mathrm{L}}$ and $\boldsymbol{y}^{\mathrm{L}}$ the unitary vectors along each axis then

\footnotetext{
${ }^{3}$ We use Eq. (A16) in Ref. [14] for the interference term. The direct terms vanish because the possible non-zero field pseudovectors are purely imaginary, e.g. $\left(\boldsymbol{E}_{3 \omega}^{*} \times \boldsymbol{E}_{3 \omega}\right)$, while the accompanying molecular pseudoscalars are real and the coupling coefficients appear within absolute values, see Ref. [38].
} 


$$
\boldsymbol{E}^{\mathrm{L}}(t)=2 F(t)\left[\cos (\omega t) \boldsymbol{x}^{\mathrm{L}}+\cos (3 \omega t+\phi) \boldsymbol{y}^{\mathrm{L}}\right]
$$

and we obtain

$$
\left\langle\boldsymbol{d}_{3}^{\mathrm{L}}\right\rangle_{\omega=0}=2 \chi_{3} \Re\left\{A_{3}^{(1) *} A_{3}^{(3)} e^{i \phi}\right\} z^{\mathrm{L}},
$$

i.e., $\left\langle\boldsymbol{d}_{3}^{\mathrm{L}}\right\rangle_{\omega=0}$ is perpendicular to the polarization plane and its magnitude and sign can be controlled through the relative phase $\phi$. Note that the relative phase of the coupling coefficients $A_{3}^{(1)}$ and $A_{3}^{(3)}$, which can be modified for example by changing the detunings, must also be taken into account.

The contributions from states $|1\rangle$, and $|2\rangle$ to the permanent dipole (5) have the same structure as Eq. (10), albeit with different coupling constants and molecular pseudoscalars $\chi_{1}$ and $\chi_{2}$, respectively [see Eq. (7)]. Since $\left|a_{0}\right|^{2}=1-\left|a_{1}\right|^{2}-\left|a_{2}\right|^{2}-$ $\left|a_{3}\right|^{2}$, the contribution from the ground state involves the coupling constants associated to $|1\rangle,|2\rangle$, and $|3\rangle$, and a molecular pseudoscalar $\chi_{0}$ [see Eq. (7)]. Together, these contributions yield

$$
\begin{aligned}
\left\langle\boldsymbol{d}^{\mathrm{L}}\right\rangle_{\omega=0}= & 2\left[\left(\chi_{1}-\chi_{0}\right) \Re\left\{A_{1}^{(1)} A_{1}^{(3) *} e^{i \phi}\right\}+\left(\chi_{2}-\chi_{0}\right) \Re\left\{A_{2}^{(2) * *} A_{2}^{(2)} e^{i \phi}\right\}\right. \\
& \left.+\left(\chi_{3}-\chi_{0}\right) \Re\left\{A_{3}^{(1) *} A_{3}^{(3)} e^{i \phi}\right\}\right] z^{\mathrm{L}}
\end{aligned}
$$

where $A_{1}^{(1)}$ and $A_{1}^{(3)}$ are the coupling coefficients for the transitions $|0\rangle \stackrel{\omega}{\rightarrow}|1\rangle$ and $|0\rangle$ $\stackrel{3 \omega}{\rightarrow}|3\rangle \stackrel{-\omega}{\rightarrow}|2\rangle \stackrel{-\omega}{\rightarrow}|1\rangle$, respectively; $A_{2}^{(2)}$ and $A_{2}^{(2) \prime}$ are the coupling coefficients for the transitions $|0\rangle \stackrel{\omega}{\rightarrow}|1\rangle \stackrel{\omega}{\rightarrow}|2\rangle$ and $|0\rangle \stackrel{3 \omega}{\rightarrow}|3\rangle \stackrel{-\omega}{\rightarrow}|2\rangle$, respectively.

In the absence of the intermediate resonances through the states $|1\rangle$ and $|2\rangle$ the contribution from the third-order term in Eq. (3) turns into a sum over all intermediate states $|j\rangle$ and $|k\rangle$ weighted by a coefficient $A_{3 ; j k}^{(3)}$. The intermediate states retain no population at the end of the pulse and the permanent dipole takes the form

$$
\begin{aligned}
\left\langle\boldsymbol{d}^{\mathrm{L}}\right\rangle_{\omega=0} & =2 \sum_{j, k}\left(\chi_{3 ; j k}-\chi_{0 ; j k}\right) \Re\left\{A_{3}^{(1) *} A_{3 ; j k}^{(3)} \boldsymbol{Z}^{\mathrm{L}}\right\} \\
& =2 F_{0}^{4}\left[2 \pi \delta_{\sigma}(\Delta)\right]^{2} \sum_{j, k} \frac{\chi_{3 ; j k}-\chi_{0 ; j k}}{\left(\omega_{k, 0}-2 \omega_{L}\right)\left(\omega_{j, 0}-\omega_{L}\right)} \Re\left\{\boldsymbol{Z}^{\mathrm{L}}\right\}
\end{aligned}
$$

which is valid for arbitrary polarizations [see Eq. (8)]. Here $\chi_{i ; j k}$ is given by Eq. (7) with the replacements $1 \rightarrow j$ and $2 \rightarrow k$. In the second equality we wrote the coupling constants explicitly, $\omega_{i, j} \equiv \omega_{i}-\omega_{j}, \Delta \equiv \omega_{3,0}-3 \omega_{L}$, and we took $\int_{-\infty}^{\infty} \mathrm{d} t F(t) e^{i \omega t} \equiv$ $2 \pi F_{0} \delta_{\sigma}(\omega)$ with $\delta_{\sigma}(\omega)$ equal to the Dirac delta in the limit of infinitesimal $\sigma$. 


\subsection{A Simple Picture of the Mechanism Leading to the Enantiosensitive Permanent Dipole}

The orientation averaging procedure we applied [38], although very powerful, is also rather formal. Below we demonstrate that the mechanism leading to the generation of the permanent dipole $\left\langle\boldsymbol{d}^{\mathrm{L}}\right\rangle_{\omega=0}$ stems from the sensitivity of the excitation to the molecular orientation and handedness, which induces uniaxial and enantiosensitive orientation of the initially isotropic sample. We remark that the excitation induces orientation $(\uparrow)$ as opposed to just alignment $(\uparrow)$ and that this orientation is furthermore enantiosensitive.

Consider the interaction of the field (9) with a dummy molecule with $\boldsymbol{d}_{1,0}^{\mathrm{M}}$ and $\boldsymbol{d}_{3,0}^{\mathrm{M}}$ perpendicular to each other and $\boldsymbol{d}_{3,2}^{\mathrm{M}}=\boldsymbol{d}_{2,1}^{\mathrm{M}}=\boldsymbol{d}_{1,0}^{\mathrm{M}}$. For simplicity we again assume that intermediate states are resonantly excited and that only state $|3\rangle$ has a non-zero permanent dipole. The population $P_{3}(\varrho) \equiv\left|a_{3}(\varrho)\right|^{2}$ of the excited state $|3\rangle$ reads [see Eq. (3)]

$$
P_{3}(\varrho)=\left|A_{3}^{(1)}\right|^{2} \mathcal{P}_{3 \omega}(\varrho)+\left|A_{3}^{(3)}\right|^{2} \mathcal{P}_{\omega}(\varrho)+2 \Re\left\{A_{3}^{(1) *} A_{3}^{(3)} e^{i \phi}\right\} \mathcal{P}_{\omega, 3 \omega}(\varrho)
$$

where

$$
\begin{gathered}
\mathcal{P}_{\omega}(\varrho) \equiv\left[\boldsymbol{d}_{1,0}^{\mathrm{L}}(\varrho) \cdot \boldsymbol{x}^{\mathrm{L}}\right]^{6}, \quad \mathcal{P}_{3 \omega}(\varrho) \equiv\left[\boldsymbol{d}_{3,0}^{\mathrm{L}}(\varrho) \cdot \boldsymbol{y}^{\mathrm{L}}\right]^{2}, \\
\mathcal{P}_{\omega, 3 \omega}(\varrho) \equiv\left[\boldsymbol{d}_{3,0}^{\mathrm{L}}(\varrho) \cdot \boldsymbol{y}^{\mathrm{L}}\right]\left[\boldsymbol{d}_{1,0}^{\mathrm{L}}(\varrho) \cdot \boldsymbol{x}^{\mathrm{L}}\right]^{3}
\end{gathered}
$$

$\mathcal{P}_{\omega}$ will select molecular orientations where $\boldsymbol{d}_{1,0}^{\mathrm{L}}$ is aligned along the $\boldsymbol{x}^{\mathrm{L}}$ axis. $\mathcal{P}_{3 \omega}$ will select molecular orientations where $\boldsymbol{d}_{3,0}^{\mathrm{L}}$ is aligned along the $\boldsymbol{y}^{\mathrm{L}}$ axis. $\mathcal{P}_{\omega, 3 \omega}$ will select molecular orientations where $\boldsymbol{d}_{1,0}^{\mathrm{L}}$ is aligned along the $\boldsymbol{x}^{\mathrm{L}}$ axis and $\boldsymbol{d}_{3,0}^{\mathrm{L}}$ is aligned along the $\boldsymbol{y}^{\mathrm{L}}$ axis. These orientations are shown in Fig. 3b. While the direct terms $\mathcal{P}_{\omega}$ and $\mathcal{P}_{3 \omega}$ do not distinguish between this subset of orientations $\left\{\varrho_{i}\right\}_{i=1}^{4}$, the interference term $\mathcal{P}_{\omega, 3 \omega}$ will be positive for orientations $\varrho_{1}$ and $\varrho_{3}$ and negative for orientations $\varrho_{2}$ and $\varrho_{4}$. This produces an imbalance between the number of excited molecules with orientations $\varrho_{1}$ and $\varrho_{3}$ and those with orientations $\varrho_{2}$ and $\varrho_{4}$. As can be seen in Fig. 3, this imbalance amounts to the molecular axis $\boldsymbol{d}_{1,0}^{\mathrm{M}} \times \boldsymbol{d}_{3,0}^{\mathrm{M}}$ being oriented. That is, the field (9) induces field-free uniaxial orientation of the molecular sample in the state $|3\rangle$. The emergence of a permanent dipole follows trivially, provided that $\boldsymbol{d}_{3,3}^{\mathrm{M}}$ has a nonzero component along the oriented axis, i.e. as long as $\boldsymbol{d}_{3,3}^{\mathrm{M}} \cdot\left(\boldsymbol{d}_{1,0}^{\mathrm{M}} \times \boldsymbol{d}_{3,0}^{\mathrm{M}}\right) \neq 0$. Note that, according to Eq. (7), this is in agreement with having $\chi_{3} \neq 0$. If we consider the situation depicted in Fig. 3 now mirror reflected across the polarization plane, which is equivalent to swapping the enantiomer while leaving the field as it is, we immediately see that $\boldsymbol{d}_{3,3}^{\mathrm{M}}$ and therefore also $\left\langle\boldsymbol{d}_{3}^{\mathrm{L}}\right\rangle_{\omega=0}$ point in the opposite direction, which explains the enantiosensitivity of $\left\langle\boldsymbol{d}_{3}^{\mathrm{L}}\right\rangle_{\omega=0}$.

Since the emergence of a permanent dipole $\left\langle\boldsymbol{d}^{\mathrm{L}}\right\rangle_{\omega=0}$ relies on the molecules in the excited state $|3\rangle$ being oriented, we expect $\left\langle\boldsymbol{d}^{\mathrm{L}}\right\rangle_{\omega=0}$ to survive for at least a few picoseconds before decaying due to molecular rotation. A decay of the dipole 


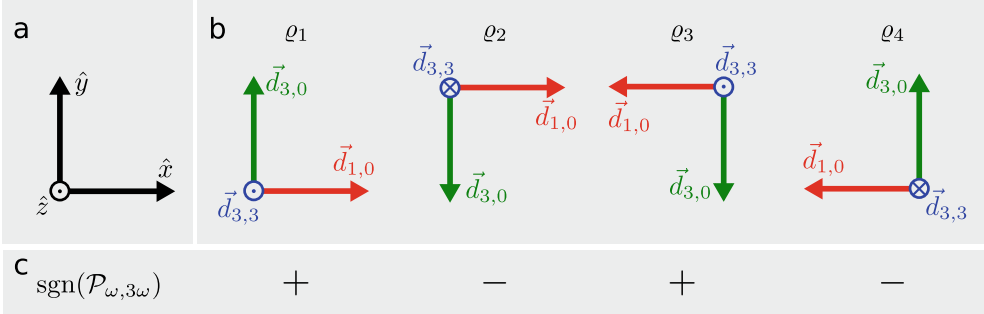

Fig. 3 Simplified analysis of the mechanism leading to an enantiosensitive permanent dipole for a field (9) and a dummy molecule with $\boldsymbol{d}_{1,0}$ and $\boldsymbol{d}_{3,0}$, perpendicular to each other and $\boldsymbol{d}_{3,2}=\boldsymbol{d}_{2,1}=$ $\boldsymbol{d}_{1,0}$. Only the component of $\boldsymbol{d}_{3,3}$ perpendicular to the plane defined by $\boldsymbol{d}_{1,0}$ and $\boldsymbol{d}_{3,0}$ is shown. a. Laboratory frame. b. Molecular orientations with $\boldsymbol{d}_{1,0}$ aligned along $\boldsymbol{x}$ and $\boldsymbol{d}_{3,0}$ aligned along $\boldsymbol{y}$. c. Sign of the interference term (15) for each molecular orientation. The interference distinguishes orientations $\varrho_{1}$ and $\varrho_{3}$ from orientations $\varrho_{2}$ and $\varrho_{4}$ and therefore causes the molecular axis $\boldsymbol{d}_{3,3}$ to become oriented. This leads to a non-vanishing permanent dipole

on the picosecond time-scale should lead to broadband $\mathrm{THz}$ emission [39] with an enantiosensitive phase. Furthermore, a quantum treatment of the rotational dynamics might reveal revivals of the molecular orientation (see e.g. Ref. [31]) .

\section{Exciting an Enantiosensitive Permanent Quadrupole}

Let us now consider the control scheme depicted in Fig. 4, where the interference of contributions from a one- $2 \omega$-photon pathway and a two- $\omega$-photon pathway control the population of the state $|2\rangle$ of a chiral molecule. In this case the field reads as

$$
\boldsymbol{E}^{\mathrm{L}}(t)=F(t)\left(\boldsymbol{E}_{\omega}^{\mathrm{L}} e^{-i \omega t}+\boldsymbol{E}_{2 \omega}^{\mathrm{L}} e^{-2 i \omega t}\right)+\text { c.c. }
$$

As in the previous section we begin assuming an intermediate resonance and then consider the case where the intermediate state is not resonant. The wave function reads as in Eq. (2) but with a sum up to $i=2$,

$$
a_{2}(\varrho)=A_{2}^{(1)}\left[\boldsymbol{d}_{2,0}^{\mathrm{L}}(\varrho) \cdot \boldsymbol{E}_{2 \omega}^{\mathrm{L}}\right]+A_{2}^{(2)}\left[\boldsymbol{d}_{2,1}^{\mathrm{L}}(\varrho) \cdot \boldsymbol{E}_{\omega}^{\mathrm{L}}\right]\left[\boldsymbol{d}_{1,0}^{\mathrm{L}}(\varrho) \cdot \boldsymbol{E}_{\omega}^{\mathrm{L}}\right] .
$$


Fig. 4 Excitation scheme used to produce an enantiosensitive permanent quadrupole in an isotropic sample of chiral molecules

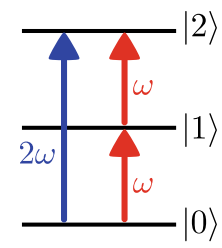

and an analogous expression for $a_{1}(\varrho)$. The expected value of the permanent electric quadrupole operator in the molecular frame $\left\langle Q_{p, q}^{\mathrm{M}}(\varrho)\right\rangle \equiv\left\langle\Psi^{\mathrm{M}}\left(\boldsymbol{r}^{\mathrm{M}}, \varrho\right)\right| Q_{p, q}^{\mathrm{M}}$ $\left|\Psi^{\mathrm{M}}\left(\boldsymbol{r}^{\mathrm{M}}, \varrho\right)\right\rangle$, where $p, q=x, y, z$, will have a zero-frequency component of the form

$$
\left\langle Q_{p, q}^{\mathrm{M}}(\varrho)\right\rangle_{\omega=0}=\sum_{i=0}^{2}\left|a_{i}(\varrho)\right|^{2}\left\langle Q_{p, q}^{\mathrm{M}}\right\rangle_{i, i}
$$

where $\left\langle Q_{p, q}^{\mathrm{M}}\right\rangle_{i, i} \equiv\left\langle\psi_{i}^{\mathrm{M}}\left|Q_{q, p}^{\mathrm{M}}\right| \psi_{i}^{\mathrm{M}}\right\rangle$. Transforming $\left\langle Q_{p, q}^{\mathrm{M}}(\varrho)\right\rangle_{\omega=0}$ to the laboratory frame and averaging over all molecular orientations yields the permanent quadrupole

$$
\left\langle Q_{p, q}^{\mathrm{L}}\right\rangle_{\omega=0} \equiv \int \mathrm{d} \varrho\left\langle Q_{p, q}^{\mathrm{L}}(\varrho)\right\rangle_{\omega=0}
$$

The contribution of state $|2\rangle$ to this expression reads as (see Appendix)

$$
\begin{aligned}
\left\langle\left(Q_{2}^{\mathrm{L}}\right)_{p, q}\right\rangle_{\omega=0} & \equiv \int \mathrm{d} \varrho\left|a_{2}(\varrho)\right|^{2}\left\langle Q_{p, q}^{\mathrm{L}}(\varrho)\right\rangle_{2,2} \\
& =\left\langle\left(Q_{2}^{\mathrm{L}}\right)_{p, q}\right\rangle_{\omega=0}^{(\text {achiral })}+\left[A_{2}^{(1) *} A_{2}^{(2)} \chi_{2}^{\prime} Z_{p, q}^{\mathrm{L}}+\text { c.c. }\right],
\end{aligned}
$$

where $\left\langle\left(Q_{2}^{\mathrm{L}}\right)_{p, q}\right\rangle_{\omega=0}^{\text {(achiral) }}$ results from the diagonal terms in $\left|a_{2}(\varrho)\right|^{2}$ and is not enantiosensitive. $\chi_{2}^{\prime}$ is a rotationally invariant molecular pseudoscalar (zero for achiral molecules) encoding the enantiosensitivity of $\left\langle\left(Q_{2}^{\mathrm{L}}\right)_{p, q}\right\rangle_{\omega=0}$ and defined according to

$$
\chi_{i}^{\prime} \equiv \frac{1}{30}\left\{\left[\left(\boldsymbol{d}_{1,0}^{\mathrm{M}} \times \boldsymbol{d}_{2,0}^{\mathrm{M}}\right) \cdot\left(\left\langle Q^{\mathrm{M}}\right\rangle_{i, i} \boldsymbol{d}_{2,1}^{\mathrm{M}}\right)\right]+\left[\left(\boldsymbol{d}_{2,1}^{\mathrm{M}} \times \boldsymbol{d}_{2,0}^{\mathrm{M}}\right) \cdot\left(\left\langle Q^{\mathrm{M}}\right\rangle_{i, i} \boldsymbol{d}_{1,0}^{\mathrm{M}}\right)\right]\right\}
$$

with $\left\langle Q^{\mathrm{M}}\right\rangle_{i, i}$ a quadrupole matrix, i.e. $\left\langle Q^{\mathrm{M}}\right\rangle_{i, i} \boldsymbol{d}_{2,1}^{\mathrm{M}}$ and $\left\langle Q^{\mathrm{M}}\right\rangle_{i, i} \boldsymbol{d}_{1,0}^{\mathrm{M}}$ denote multiplications of a matrix and a vector. $Z_{p, q}^{\mathrm{L}}$ is a symmetric field pseudotensor of rank 2 . It encodes the dependence of $\left\langle\left(Q_{2}^{\mathrm{L}}\right)_{p, q}\right\rangle_{\omega=0}$ on the field polarization according to

$$
Z_{p, q}^{\mathrm{L}} \equiv\left(\boldsymbol{E}_{\omega}^{\mathrm{L}} \times \boldsymbol{E}_{2 \omega}^{\mathrm{L} *}\right)_{p}\left(\boldsymbol{E}_{\omega}^{\mathrm{L}}\right)_{q}+\left(\boldsymbol{E}_{\omega}^{\mathrm{L}} \times \boldsymbol{E}_{2 \omega}^{\mathrm{L} *}\right)_{q}\left(\boldsymbol{E}_{\omega}^{\mathrm{L}}\right)_{p}
$$

This expression shows that all components of $Z_{p, q}^{\mathrm{L}}$ vanish if $\omega$ and $2 \omega$ are linearly polarized parallel to each other, or if $\omega$ and $2 \omega$ are circularly polarized and counterrotating. 
For example, if we take $\omega$ and $2 \omega$ linearly polarized perpendicular to each other, say $\boldsymbol{E}_{\omega}^{\mathrm{L}}=\boldsymbol{x}^{\mathrm{L}}$ and $\boldsymbol{E}_{2 \omega}^{\mathrm{L}}=e^{-i \phi} \boldsymbol{y}^{\mathrm{L}}$, then

$$
\boldsymbol{E}^{\mathrm{L}}(t)=2 F(t)\left[\cos (\omega t) \boldsymbol{x}^{\mathrm{L}}+\cos (2 \omega t+\phi) \boldsymbol{y}^{\mathrm{L}}\right]
$$

and we obtain

$$
\left\langle\left(Q_{2}^{\mathrm{L}}\right)_{p, q}\right\rangle_{\omega=0}=\left\langle\left(Q_{2}^{\mathrm{L}}\right)_{p, q}\right\rangle_{\omega=0}^{(\text {achiral })}+2 \chi_{2}^{\prime} \Re\left\{A_{2}^{(1) *} A_{2}^{(2)} e^{i \phi}\right\}\left(\delta_{p, z} \delta_{q, x}+\delta_{q, z} \delta_{p, x}\right) .
$$

Furthermore, one can show that for the field (24) the achiral terms vanish for $p \neq q$ (see Appendix) and therefore the enantiosensitive $x z$ component reads as

$$
\left\langle\left(Q_{2}^{\mathrm{L}}\right)_{x, z}\right\rangle_{\omega=0}=2 \chi_{2}^{\prime} \Re\left\{A_{2}^{(1) *} A_{2}^{(2)} e^{i \phi}\right\}
$$

i.e., it doesn't have an achiral background and can be controlled through the relative phase $\phi$. The other non-diagonal components $x y$ and $y z$ vanish.

The contribution from state $|1\rangle$ to the permanent quadrupole (19) has the same structure as Eq. (26), although with different coupling constants and molecular pseudoscalar $\chi_{1}^{\prime}$ [see Eq. (22)]. Since $\left|a_{0}\right|^{2}=1-\left|a_{1}\right|^{2}-\left|a_{2}\right|^{2}$, the contribution from the ground state involves the coupling constants associated to $|1\rangle$ and $|2\rangle$, and a molecular pseudoscalar $\chi_{0}^{\prime}$ [see Eq. (22)]. Together, these contributions yield

$$
\left\langle Q_{x, z}^{\mathrm{L}}\right\rangle_{\omega=0}=2\left[\left(\chi_{1}^{\prime}-\chi_{0}^{\prime}\right) \Re\left\{A_{1}^{(1)} A_{1}^{(2) *} e^{i \phi}\right\}+\left(\chi_{2}^{\prime}-\chi_{0}^{\prime}\right) \Re\left\{A_{2}^{(1) *} A_{2}^{(2)} e^{i \phi}\right\}\right],
$$

where $A_{1}^{(1)}$ and $A_{1}^{(2)}$ are the coupling coefficients for the transitions $|0\rangle \stackrel{\omega}{\rightarrow}|1\rangle$ and $|0\rangle \stackrel{2 \omega}{\rightarrow}|2\rangle \stackrel{-\omega}{\rightarrow}|1\rangle$, respectively. The other non-diagonal elements of the permanent quadrupole vanish and the diagonal terms are not enantiosensitive.

As in the previous section, in the absence of an intermediate resonance through the state $|1\rangle$, the contribution from the second-order term in Eq. (17) turns into a sum over all intermediate states $|j\rangle$. The intermediate states retain no population at the end of the pulse and the permanent quadrupole takes the form

$$
\begin{aligned}
\left\langle Q_{x, z}^{\mathrm{L}}\right\rangle_{\omega=0} & =2 \sum_{j}\left(\chi_{2 ; j}^{\prime}-\chi_{0 ; j}^{\prime}\right) \Re\left\{A_{2}^{(1) *} A_{2 ; j}^{(2)} e^{i \phi}\right\} \\
& =2 F_{0}^{3}\left[2 \pi \delta_{\sigma}(\Delta)\right]^{2} \sum_{j} \frac{\chi_{2 ; j}^{\prime}-\chi_{0 ; j}^{\prime}}{\omega_{j, 0}-\omega_{L}} \cos \phi,
\end{aligned}
$$

where $\Delta \equiv \omega_{2,0}-2 \omega_{L}, \chi_{i ; j}$ is given by Eq. (22) with the replacement $1 \rightarrow j$ and the other symbols were introduced as in Eq. (12).

A simplified analysis analogous to that presented in Fig. 3 is shown in Fig. 5 for the case of the field (24) interacting with a dummy molecule with $\boldsymbol{d}_{1,0}^{\mathrm{M}}, \boldsymbol{d}_{2,0}^{\mathrm{M}}$, and $\left\langle Q^{\mathrm{M}}\right\rangle_{2,2}$ oriented as shown and with $\boldsymbol{d}_{2,1}^{\mathrm{M}}=\boldsymbol{d}_{1,0}^{\mathrm{M}}$. The population of state $|2\rangle$ is determined by 


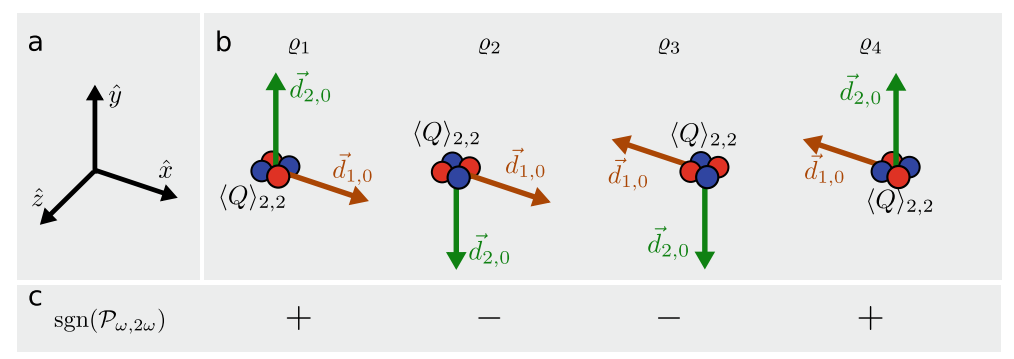

Fig. 5 Simplified analysis of the mechanism leading to an enantiosensitive permanent quadrupole for a field (24) and a dummy molecule with $\boldsymbol{d}_{1,0}, \boldsymbol{d}_{2,0}$, and $\langle Q\rangle_{2,2}$ oriented as shown with respect to each other and $\boldsymbol{d}_{2,1}=\boldsymbol{d}_{1,0}$. Blue and red balls stand for negative and positive charges. a. Laboratory frame. b. Molecular orientations with $\boldsymbol{d}_{1,0}$ aligned along $\boldsymbol{x}$ and $\boldsymbol{d}_{2,0}$ aligned along $\boldsymbol{y}$. c. Sign of the interference term (29) for each molecular orientation. The interference causes the molecular axis $\boldsymbol{d}_{2,0}$ to become oriented, which together with the alignment of $\boldsymbol{d}_{1,0}$ along $\boldsymbol{x}$ explains the non-vanishing permanent quadrupole

the interference term

$$
\mathcal{P}_{\omega, 2 \omega}(\varrho) \equiv\left[\boldsymbol{d}_{2,0}(\varrho) \cdot \boldsymbol{y}\right]\left[\boldsymbol{d}_{1,0}(\varrho) \cdot \boldsymbol{x}\right]^{2},
$$

which is positive for orientations $\varrho_{1}$ and $\varrho_{4}$ and negative for orientations $\varrho_{2}$ and $\varrho_{3}$. This causes the molecular axis $\boldsymbol{d}_{2,0}^{\mathrm{M}}$ to become oriented. If $\boldsymbol{d}_{2,2}^{\mathrm{M}}$ has a non-zero component along $\boldsymbol{d}_{2,0}^{\mathrm{M}}$, then a permanent dipole emerges. However, this permanent dipole is contained in the polarization plane and will therefore not change upon reflection of the system across the polarization plane. Since this reflection is equivalent to a change of the enantiomer, the permanent dipole is not enantiosensitive. In contrast and as can be seen from Fig. 5, the imbalance between orientations $\varrho_{1}$ and $\varrho_{4}$ in comparison to orientations $\varrho_{2}$ and $\varrho_{3}$ is enough to produce an enantiosensitive permanent quadrupole that does change upon reflection in the polarization plane.

\section{Conclusions}

We have shown that permanent dipoles and quadrupoles can be induced in initially isotropic samples of chiral molecules using perturbative two-color fields that resonantly excite electronic transitions. These permanent multipoles are enantiosensitive and their sign can be controlled through the relative phase between the two colors. The mechanism leading to these permanent dipoles (or quadrupoles) stems from uniaxial orientation of the molecule, which occurs due to the selectivity of the excitation to the orientation of the molecule. Such orienting excitation can be accomplished using fields where the fundamental and its second (or third) harmonic are linearly polarized perpendicular to each other. The enantiosensitive permanent dipole is obtained via three- $\omega$ - versus one- $3 \omega$-photon interference. The enantiosensitive quadrupole is 
obtained via two- $\omega$ versus one- $2 \omega$ interference. In the latter case, a permanent dipole can also be generated but it is not enantiosensitive. We expect these permanent multipoles to survive for at least a few picoseconds before decaying due to molecular rotation. Such picosecond variation of the multipoles should in principle lead to broadband $\mathrm{THz}$ emission with an enantiosensitive phase.

Although we focused on a mechanism relying on interference between two pathways, it is also possible to induce permanent dipoles via direct pathways by relying on transitions where the photon order matters. This can be achieved e.g. using the pulse sequence in Ref. [29].

Efficient generation of enantio-sensitive permanent dipoles and quadrupoles via orientation-sensitive excitations is possible in strong laser fields using efficient excitation of Rydberg states via the so-called Freeman resonances [40] in the regime when the pronderomotive potential is comparable to the laser frequency. Since Rydberg states have large polarizability, we expect significant contrast in the orientation of left and right enantiomers. Opposite orientation of left and right enantiomers and their respective induced permanent dipoles create opportunities for enantio separation using static electric fields.

Acknowledgements We gratefully acknowledge support from the DFG SPP 1840 "Quantum Dynamics in Tailored Intense Fields" within the project SM 292/5-1;

\section{Appendix}

\section{Coupling coefficients $A_{f}^{(n)}$}

Consider a Hamiltonian $H=H_{0}+H^{\prime}(t)$, where $H_{0}$ is the time-independent fieldfree Hamiltonian and $H^{\prime}(t)$ can be treated as a perturbation. If at time $t=0$ the system is in the state $|0\rangle$, the probability amplitude of finding the system in the state $|f\rangle$ at the time $t=T$ can be written as $a_{f}=a_{f}^{(1)}+a_{f}^{(2)}+\cdots$, where

$$
a_{f}^{(N)}=\left(\frac{1}{i}\right)^{N} \int_{0}^{T} \mathrm{~d} t_{N} \ldots \int_{0}^{t_{3}} \mathrm{~d} t_{2} \int_{0}^{t_{2}} \mathrm{~d} t_{1}\left\langle f\left|H_{I}^{\prime}\left(t_{N}\right) \ldots H_{I}^{\prime}\left(t_{2}\right) H_{I}^{\prime}\left(t_{1}\right)\right| 0\right\rangle
$$

and $H_{I}^{\prime}(t)=e^{i H_{0} t} H^{\prime}(t) e^{-i H_{0} t}$. In the electric dipole approximation we have $H^{\prime}=$ $-\boldsymbol{d} \cdot \boldsymbol{E}(t)$. For a field $\boldsymbol{E}(t)=F(t) \boldsymbol{E}_{\omega} e^{-i \omega t}+$ c.c., the contributions to $a_{f}^{(N)}$ from absorption of $N$ photons yield

$$
a_{f}^{(N)}=\sum_{j_{1}, j_{2}, \ldots, j_{N-1}} a_{f ; j_{1}, j_{2}, \ldots, j_{N-1}}^{(N)},
$$


where the sum is over the different quantum pathways through the intermediate states $\left|j_{1}\right\rangle,\left|j_{2}\right\rangle, \ldots,\left|j_{N-1}\right\rangle$. The amplitude of each pathway can be written as

$$
a_{f ; j_{1}, j_{2} \ldots, j_{N-1}}^{(N)}=A_{f ; j_{1}, j_{2}, \ldots, j_{N-1}}^{(N)}(\omega)\left(\boldsymbol{d}_{f, j_{N-1}} \cdot \boldsymbol{E}_{\omega}\right) \ldots\left(\boldsymbol{d}_{j_{2}, j_{1}} \cdot \boldsymbol{E}_{\omega}\right)\left(\boldsymbol{d}_{j_{1}, 0} \cdot \boldsymbol{E}_{\omega}\right)
$$

The coupling coefficient $A_{f ; j_{1}, j_{2}, \ldots, j_{N-1}}^{(N)}(\omega)$ carries the information about the frequency of the light, its envelope, and the detunings according to

$$
\begin{aligned}
A_{f ; j_{1}, j_{2}, \ldots, j_{N-1}}^{(N)}(\omega) & =i^{N} \int_{0}^{T} \mathrm{~d} t_{N} F\left(t_{N}\right) e^{i\left(\omega_{f, j_{N-1}}-\omega\right) t_{N}} \ldots \\
& \times \int_{0}^{t_{3}} \mathrm{~d} t_{2} F\left(t_{2}\right) e^{i\left(\omega_{j_{2}, j_{1}}-\omega\right) t_{2}} \int_{0}^{t_{2}} \mathrm{~d} t_{1} F\left(t_{1}\right) e^{i\left(\omega_{j_{1}, 0}-\omega\right) t_{1}},
\end{aligned}
$$

where $\omega_{i j} \equiv \omega_{i}-\omega_{j}$. Contributions to $a_{f}^{(N)}$ from pathways involving photon emissions require exchanging $\boldsymbol{E}_{\omega}$ by $\boldsymbol{E}_{\omega}^{*}$ in Eq. (32) ${ }^{4}$ and $\omega$ by $-\omega$ in Eq. (33) in the corresponding transitions.

In the case of a resonant pathway the sum in Eq. (31) reduces to a single term, which is the assumption in several parts of the main text. There we write $A_{f}^{(N)}$ as a shorthand for $A_{f ; j_{1}, j_{2}, \ldots, j_{N-1}}^{(N)}$.

\section{Orientation integrals required in Sect. 3}

Replacing Eq. (17) in Eq. (20) we obtain

$$
\left\langle Q_{p, q}^{\mathrm{L}}\right\rangle_{\omega=0}=\left|A_{2}^{(1)}\right|^{2} I_{p, q}^{(2 \omega)}+\left|A_{2}^{(2)}\right|^{2} I_{p, q}^{(\omega)}+\left[A_{2}^{(1) *} A_{2}^{(2)} I_{p, q}^{(\omega, 2 \omega)}+\text { c.c. }\right],
$$

where the integrals $I_{p, q}^{(2 \omega)}, I_{p, q}^{(\omega)}$, and $I_{p, q}^{(\omega, 2 \omega)}$ are defined by

$$
\begin{gathered}
I_{p, q}^{(2 \omega)} \equiv \int \mathrm{d} \varrho\left|\boldsymbol{d}_{2,0}^{\mathrm{L}}(\varrho) \cdot \boldsymbol{E}_{2 \omega}^{\mathrm{L}}\right|^{2}\left\langle Q_{p, q}^{\mathrm{L}}\right\rangle_{2,2}, \\
I_{p, q}^{(\omega)} \equiv \int \mathrm{d} \varrho\left|\left[\boldsymbol{d}_{2,1}^{\mathrm{L}}(\varrho) \cdot \boldsymbol{E}_{\omega}^{\mathrm{L}}\right]\left[\boldsymbol{d}_{1,0}^{\mathrm{L}}(\varrho) \cdot \boldsymbol{E}_{\omega}^{\mathrm{L}}\right]\right|^{2}\left\langle Q_{p, q}^{\mathrm{L}}\right\rangle_{2,2}, \\
I_{p, q}^{(\omega, 2 \omega)} \equiv \int \mathrm{d} \varrho\left[\boldsymbol{d}_{2,1}^{\mathrm{L}}(\varrho) \cdot \boldsymbol{E}_{\omega}^{\mathrm{L}}\right]\left[\boldsymbol{d}_{1,0}^{\mathrm{L}}(\varrho) \cdot \boldsymbol{E}_{\omega}^{\mathrm{L}}\right]\left[\boldsymbol{d}_{2,0}^{\mathrm{L}}(\varrho) \cdot \boldsymbol{E}_{2 \omega}^{\mathrm{L} *}\right]\left\langle Q_{p, q}^{\mathrm{L}}\right\rangle_{2,2} \cdot
\end{gathered}
$$

\footnotetext{
${ }^{4}$ If the transition dipoles are complex then one must also complex conjugate them. Here we assume they are real.
} 
These integrals can be solved following the procedure in Ref. [38]. We will first solve $I_{p, q}^{(\omega, 2 \omega)}$ for arbitrary polarizations and then show that $I_{p, q}^{(2 \omega)}$ and $I_{p, q}^{(\omega)}$ vanish when $p \neq q, \boldsymbol{E}_{\omega}^{\mathrm{L}}=\boldsymbol{x}$, and $\boldsymbol{E}_{2 \omega}^{\mathrm{L}}=e^{-i \phi} \boldsymbol{y}^{\mathrm{L}}$.

$I_{p, q}^{(\omega, 2 \omega)}$

We are dealing with an integral of the form

$$
\begin{aligned}
I_{i_{4} i_{5}} & =\int \mathrm{d} \varrho\left(\boldsymbol{a}^{\mathrm{L}} \cdot \boldsymbol{B}^{\mathrm{L}}\right)\left(\boldsymbol{b}^{\mathrm{L}} \cdot \boldsymbol{B}^{\mathrm{L}}\right)\left(\boldsymbol{c}^{\mathrm{L}} \cdot \boldsymbol{C}^{\mathrm{L}}\right) Q_{i_{4}, i_{5}}^{\mathrm{L}} \\
& =I_{i_{1} i_{2} i_{3} i_{4} i_{5} ; \lambda_{1} \lambda_{2} \lambda_{3} \lambda_{4} \lambda_{5}}^{(5)} a_{\lambda_{1}}^{\mathrm{M}} b_{\lambda_{2}}^{\mathrm{M}} c_{\lambda_{3}}^{\mathrm{M}} Q_{\lambda_{4}, \lambda_{5}}^{\mathrm{M}} B_{i_{1}}^{\mathrm{L}} B_{i_{2}}^{\mathrm{L}} C_{i_{3}}^{\mathrm{L}}
\end{aligned}
$$

where

$$
I_{i_{1} i_{2} i_{3} i_{4} i_{5} ; \lambda_{1} \lambda_{2} \lambda_{3} \lambda_{4} \lambda_{5}}^{(5)} \equiv \int \mathrm{d} \varrho l_{i_{1} \lambda_{1}} l_{i_{2} \lambda_{2}} l_{i_{3} \lambda_{3}} l_{i_{4} \lambda_{4}} l_{i_{5} \lambda_{5}},
$$

$\boldsymbol{a}^{\mathrm{M}}, \boldsymbol{b}^{\mathrm{M}}$, and $\boldsymbol{c}^{\mathrm{M}}$ are arbitrary vectors fixed in the molecular frame, and $Q_{i_{4}, i_{5}}^{\mathrm{M}}$ is an arbitrary symmetric second-rank tensor fixed in the molecular frame. The transformation to the laboratory frame is given by $v_{i}^{\mathrm{L}}(\varrho)=l_{i \lambda}(\varrho) v_{\lambda}^{\mathrm{M}}$ for vectors and $Q_{i_{1}, i_{2}}^{\mathrm{L}}(\varrho)=l_{i_{1} \lambda_{1}}(\varrho) l_{i_{2} \lambda_{2}}(\varrho) Q_{\lambda_{1}, \lambda_{2}}^{\mathrm{M}}$ for the second-rank tensor, where $l_{i \lambda}(\varrho)$ is the matrix of direction cosines, we sum over repeated indices and use latin indices for components in the laboratory frame and greek indices for components in the molecular frame. $\boldsymbol{B}^{\mathrm{L}}$ and $\boldsymbol{C}^{\mathrm{L}}$ are arbitrary vectors fixed in the laboratory frame. Using Eq. (31) in Ref. [38] we obtain

$$
\begin{aligned}
I_{i_{4} i_{5}}= & \frac{1}{30}\left[\epsilon_{\lambda_{1} \lambda_{3} \lambda_{4}} \delta_{\lambda_{2} \lambda_{5}} \epsilon_{i_{1} i_{3} i_{4}} \delta_{i_{2} i_{5}}+\epsilon_{\lambda_{1} \lambda_{3} \lambda_{5}} \delta_{\lambda_{2} \lambda_{4}} \epsilon_{i_{1} i_{3} i_{5}} \delta_{i_{2} i_{4}}+\epsilon_{\lambda_{2} \lambda_{3} \lambda_{4}} \delta_{\lambda_{1} \lambda_{5}} \epsilon_{i_{2} i_{3} i_{4}} \delta_{i_{1} i_{5}}\right. \\
& \left.+\epsilon_{\lambda_{2} \lambda_{3} \lambda_{5}} \delta_{\lambda_{1} \lambda_{4}} \epsilon_{i_{2} i_{3} i_{5}} \delta_{i_{1} i_{4}}\right] a_{\lambda_{1}}^{\mathrm{M}} b_{\lambda_{2}}^{\mathrm{M}} c_{\lambda_{3}}^{\mathrm{M}} Q_{\lambda_{4}, \lambda_{5}}^{\mathrm{M}} B_{i_{1}}^{\mathrm{L}} B_{i_{2}}^{\mathrm{L}} C_{i_{3}}^{\mathrm{L}}
\end{aligned}
$$

where we used $\epsilon_{i_{1} i_{2} i_{3}} B_{i_{2}} B_{i_{3}}=\epsilon_{\lambda_{1} \lambda_{2} \lambda_{3}} Q_{\lambda_{2} \lambda_{3}}=0$. The first term can be rewritten as

$$
\begin{aligned}
\epsilon_{\lambda_{1} \lambda_{3} \lambda_{4}} \delta_{\lambda_{2} \lambda_{5}} \epsilon_{i_{1} i_{3} i_{4}} \delta_{i_{2} i_{5}} a_{\lambda_{1}}^{\mathrm{M}} b_{\lambda_{2}}^{\mathrm{M}} c_{\lambda_{3}}^{\mathrm{M}} Q_{\lambda_{4}, \lambda_{5}}^{\mathrm{M}} B_{i_{1}}^{\mathrm{L}} B_{i_{2}}^{\mathrm{L}} C_{i_{3}}^{\mathrm{L}} \\
=\left(\boldsymbol{a}^{\mathrm{M}} \times \boldsymbol{c}^{\mathrm{M}}\right)_{\lambda_{4}} Q_{\lambda_{4}, \lambda_{5}}^{\mathrm{M}} b_{\lambda_{5}}^{\mathrm{M}}\left(\boldsymbol{B}^{\mathrm{L}} \times \boldsymbol{C}^{\mathrm{L}}\right)_{i_{4}} B_{i_{5}}^{\mathrm{L}} \\
=\left[\left(\boldsymbol{a}^{\mathrm{M}} \times \boldsymbol{c}^{\mathrm{M}}\right) \cdot\left(Q^{\mathrm{M}} \boldsymbol{b}^{\mathrm{M}}\right)\right]\left(\boldsymbol{B}^{\mathrm{L}} \times \boldsymbol{C}^{\mathrm{L}}\right)_{i_{4}} B_{i_{5}}^{\mathrm{L}} .
\end{aligned}
$$


Analogous operations for the rest of the terms yield

$$
\begin{aligned}
I_{i_{4} i_{5}}= & \frac{1}{30}\left\{\left[\left(\boldsymbol{a}^{\mathrm{M}} \times \boldsymbol{c}^{\mathrm{M}}\right) \cdot\left(Q^{\mathrm{M}} \boldsymbol{b}^{\mathrm{M}}\right)\right]+\left[\left(\boldsymbol{b}^{\mathrm{M}} \times \boldsymbol{c}^{\mathrm{M}}\right) \cdot\left(Q^{\mathrm{M}} \boldsymbol{a}^{\mathrm{M}}\right)\right]\right\} \\
& \times\left\{\left(\boldsymbol{B}^{\mathrm{L}} \times \boldsymbol{C}^{\mathrm{L}}\right)_{i_{4}} B_{i_{5}}^{\mathrm{L}}+\left(\boldsymbol{B}^{\mathrm{L}} \times \boldsymbol{C}^{\mathrm{L}}\right)_{i_{5}} B_{i_{4}}^{\mathrm{L}}\right\}
\end{aligned}
$$

Performing the substitutions $\{\boldsymbol{a}, \boldsymbol{b}, \boldsymbol{c}, Q\} \rightarrow\left\{\boldsymbol{d}_{2,1}, \boldsymbol{d}_{1,0}, \boldsymbol{d}_{2,0},\langle Q\rangle_{2,2}\right\},\{\boldsymbol{B}, \boldsymbol{C}\} \rightarrow\left\{\boldsymbol{E}_{\omega}\right.$, $\left.\boldsymbol{E}_{2 \omega}^{*}\right\}$, and $\left\{i_{4}, i_{5}\right\} \rightarrow\{p, q\}$ and using Eqs. (34) and (37) yields Eqs. (21)-(23).

$I_{p, q}^{(2 \omega)}$

Assuming a linearly polarized $\boldsymbol{E}_{2 \omega}$ we must deal with an integral of the form

$$
\begin{aligned}
I_{i_{3} i_{4}} & =\int \mathrm{d} \varrho\left[\boldsymbol{a}^{\mathrm{L}} \cdot \boldsymbol{B}^{\mathrm{L}}\right]\left[\boldsymbol{a}^{\mathrm{L}} \cdot \boldsymbol{B}^{\mathrm{L}}\right] Q_{i_{3} i_{4}}^{\mathrm{L}} \\
& =I_{i_{1} i_{2} i_{3} i_{4} ; \lambda_{1} \lambda_{2} \lambda_{3} \lambda_{4}}^{(4)} a_{\lambda_{1}}^{\mathrm{M}} a_{\lambda_{2}}^{\mathrm{M}} Q_{\lambda_{3}, \lambda_{4}}^{\mathrm{M}} B_{i_{1}}^{\mathrm{L}} B_{i_{2}}^{\mathrm{L}},
\end{aligned}
$$

where

$$
I_{i_{1} i_{2} i_{3} i_{4} ; \lambda_{1} \lambda_{2} \lambda_{3} \lambda_{4}}^{(4)} \equiv \int \mathrm{d} \varrho l_{i_{1} \lambda_{1}} l_{i_{2} \lambda_{2}} l_{i_{3} \lambda_{3}} l_{i_{4} \lambda_{4}},
$$

and we use the same notation as in the previous subsection. Using Eq. (19) in Ref. [38] we get

$$
I_{i_{3} i_{4}}=\boldsymbol{F}_{i_{3} i_{4}}^{(4)} \cdot M^{(4)} \boldsymbol{G}_{i_{3} i_{4}}^{(4)},
$$

where $\boldsymbol{F}_{i_{3} i_{4}}^{(4)}$ is given by

$$
\boldsymbol{F}_{i_{3} i_{4}}^{(4)}=\left(\begin{array}{c}
\delta_{i_{1} i_{2}} \delta_{i_{3} i_{4}} \\
\delta_{i_{1} i_{3}} \delta_{i_{2} i_{4}} \\
\delta_{i_{1} i_{4}} \delta_{i_{2} i_{3}}
\end{array}\right) B_{i_{1}}^{\mathrm{L}} B_{i_{2}}^{\mathrm{L}}=\left(\begin{array}{c}
\left|\boldsymbol{B}^{\mathrm{L}}\right|^{2} \delta_{i_{3} i_{4}} \\
B_{i_{3}}^{\mathrm{L}} B_{i_{4}}^{\mathrm{L}} \\
B_{i_{3}}^{\mathrm{L}} B_{i_{4}}^{\mathrm{L}}
\end{array}\right)
$$

For $\boldsymbol{B}^{\mathrm{L}}=\boldsymbol{y}^{\mathrm{L}}$ we have $B_{i}^{\mathrm{L}}=\delta_{i y}$ and therefore $B_{i_{3}}^{\mathrm{L}} B_{i_{4}}^{\mathrm{L}}=\delta_{i_{3} y} \delta_{i_{4} y}=\delta_{i_{3} i_{4}} \delta_{i_{3} y}$, which yields $\boldsymbol{F}_{i_{3} i_{4}}^{(4)} \propto \delta_{i_{3} i_{4}} \quad$ and $I_{i_{3} i_{4}} \propto \delta_{i_{3} i_{4}}$. The substitutions $\quad\left\{\boldsymbol{a}, Q, \boldsymbol{B}, i_{3}, i_{4}\right\} \rightarrow\left\{\boldsymbol{d}_{2,0}\right.$, $\left.\langle Q\rangle_{2,2}, \boldsymbol{y}, p, q\right\}$ then yield $I_{p, q}^{(2 \omega)} \propto \delta_{p, q}$.

$I_{p, q}^{(\omega)}$

Assuming a linearly polarized $\boldsymbol{E}_{\omega}$ we must deal with an integral of the form 


$$
\begin{aligned}
I_{i_{5}, i_{6}} & =\int \mathrm{d} \varrho\left[\boldsymbol{a}^{\mathrm{L}} \cdot \boldsymbol{B}^{\mathrm{L}}\right]\left[\boldsymbol{b}^{\mathrm{L}} \cdot \boldsymbol{B}^{\mathrm{L}}\right]\left[\boldsymbol{a}^{\mathrm{L}} \cdot \boldsymbol{B}^{\mathrm{L}}\right]\left[\boldsymbol{b}^{\mathrm{L}} \cdot \boldsymbol{B}^{\mathrm{L}}\right] Q_{i_{5} i_{6}}^{\mathrm{L}} \\
& =I_{i_{1} i_{2} i_{3} i_{4} i_{5} i_{6} ; \lambda_{1} \lambda_{2} \lambda_{3} \lambda_{4} \lambda_{5} \lambda_{6}}^{(6)} a_{\lambda_{1}}^{\mathrm{M}} b_{\lambda_{2}}^{\mathrm{M}} a_{\lambda_{3}}^{\mathrm{M}} b_{\lambda_{4}}^{\mathrm{M}} Q_{\lambda_{5}, \lambda_{6}}^{\mathrm{M}} B_{i_{1}}^{\mathrm{L}} B_{i_{2}}^{\mathrm{L}} B_{i_{3}}^{\mathrm{L}} B_{i_{4}}^{\mathrm{L}},
\end{aligned}
$$

where

$$
I_{i_{1} i_{2} i_{3} i_{4} i_{5} i_{6} ; \lambda_{1} \lambda_{2} \lambda_{3} \lambda_{4} \lambda_{5} \lambda_{6}}^{(6)}=\int \mathrm{d} \varrho l_{i_{1} \lambda_{1}} l_{i_{2} \lambda_{2}} l_{i_{3} \lambda_{3}} l_{i_{4} \lambda_{4}} l_{i_{5} \lambda_{5}} l_{i_{6} \lambda_{6}}
$$

and we use the same notation as in the previous subsections. Using Table II in [38] we have that

$$
I_{i_{5}, i_{6}}=\boldsymbol{F}_{i_{5} i_{6}}^{(6)} \cdot M^{(6)} \boldsymbol{G}_{i_{3} i_{4}}^{(6)},
$$

where $\left(F_{i_{5} i_{6}}^{(6)}\right)_{r} \equiv f_{r}^{(6)} B_{i_{1}}^{\mathrm{L}} B_{i_{2}}^{\mathrm{L}} B_{i_{3}}^{\mathrm{L}} B_{i_{4}}^{\mathrm{L}}$ and $f_{r}^{(6)}(r=1,2, \ldots, 15)$ is given in Table II in [38]. For $\boldsymbol{B}^{\mathrm{L}}=\boldsymbol{x}^{\mathrm{L}}$ we have $B_{i}^{\mathrm{L}}=\delta_{i x}$ and therefore

$$
\left(F_{i_{5} i_{6}}^{(6)}\right)_{r}= \begin{cases}\delta_{i_{5} i_{6}}, & r=1,4,7 \\ \delta_{i_{5} x} \delta_{i_{6} x}, & \text { otherwise }\end{cases}
$$

Since $\delta_{i_{5} x} \delta_{i_{6} x}=\delta_{i_{5} i_{6}} \delta_{i_{5} x}$, then $\boldsymbol{F}_{i_{5} i_{6}}^{(6)} \propto \delta_{i_{5} i_{6}}$ and $I_{i_{5}, i_{6}} \propto \delta_{i_{5} i_{6}}$. The substitutions $\{\boldsymbol{a}, \boldsymbol{b}, Q$, $\left.\boldsymbol{B}, i_{5}, i_{6}\right\} \rightarrow\left\{\boldsymbol{d}_{1,0}, \boldsymbol{d}_{2,1},\langle Q\rangle_{2,2}, \boldsymbol{x}, p, q\right\}$ then yield $I_{p, q}^{(\omega)} \propto \delta_{p, q}$.

\section{References}

1. E.U. Condon, Rev. Mod. Phys. 9(4), 432 (1937). https://doi.org/10.1103/RevModPhys.9.432. URL https://link.aps.org/doi/10.1103/RevModPhys.9.432

2. K. Soai, T. Shibata, H. Morioka, K. Choji, Nature 378(6559), 767 (1995). https://doi.org/10. 1038/378767a0. URL https://www.nature.com/articles/378767a0. Number: 6559 Publisher: Nature Publishing Group

3. P. Fischer, F. Hache, Chirality 17(8), 421 (2005). https://doi.org/10.1002/chir.20179. URL https://onlinelibrary.wiley.com/doi/abs/10.1002/chir.20179

4. S. Beaulieu, A. Ferré, R. Géneaux, R. Canonge, D. Descamps, B. Fabre, N. Fedorov, F. Légaré, S. Petit, T. Ruchon, V. Blanchet, Y. Mairesse, B. Pons, New J. Phys. 18(10), 102002 (2016). https://doi.org/10.1088/1367-2630/18/10/102002. URL http://stacks.iop.org/1367-2630/18/ $\mathrm{i}=10 / \mathrm{a}=102002$

5. K. Banerjee-Ghosh, O.B. Dor, F. Tassinari, E. Capua, S. Yochelis, A. Capua, S.H. Yang, S.S.P. Parkin, S. Sarkar, L. Kronik, L.T. Baczewski, R. Naaman, Y. Paltiel, Science 360(6395), 1331 (2018). https://doi.org/10.1126/science.aar4265. URL https://science.sciencemag.org/ content/360/6395/1331. Publisher: American Association for the Advancement of Science Section: Report

6. D.S. Sanchez, I. Belopolski, T.A. Cochran, X. Xu, J.X. Yin, G. Chang, W. Xie, K. Manna, V. Süß, C.Y. Huang, N. Alidoust, D. Multer, S.S. Zhang, N. Shumiya, X. Wang, G.Q. Wang, T.R. Chang, C. Felser, S.Y. Xu, S. Jia, H. Lin, M.Z. Hasan, Nature 567(7749), 500 (2019). https://doi. org/10.1038/s41586-019-1037-2. URL https://www.nature.com/articles/s41586-019-1037-2

7. S. Mason, Chemical Society Reviews 17, 347 (1988). https://doi.org/10.1039/CS9881700347. URL https://pubs.rsc.org/en/content/articlelanding/1988/cs/cs9881700347 
8. D.G. Blackmond, Proc. Nat. Acad. Sci. 101(16), 5732 (2004). https://doi.org/10.1073/pnas. 0308363101. URL https://www.pnas.org/content/101/16/5732

9. G.Q. Lin, Q.D. You, J.F. Cheng, Chiral Drugs: Chemistry and Biological Action (Wiley, Hoboken, NJ, 2011)

10. N. Berova, P.L. Polavarapu, K. Nakanishi, R.W. Woody, Comprehensive Chiroptical Spectroscopy, vol. 1 (Wiley, Hoboken, NJ, 2012)

11. B. Ritchie, Phys. Rev. A 13(4), 1411 (1976). URL http://journals.aps.org/pra/abstract/10.1103/ PhysRevA.13.1411

12. N. Böwering, T. Lischke, B. Schmidtke, N. Müller, T. Khalil, U. Heinzmann, Phys. Rev. Lett. 86(7), 1187 (2001). https://doi.org/10.1103/PhysRevLett.86.1187. URL http://link.aps. org/doi/10.1103/PhysRevLett.86.1187

13. I. Powis, in Advances in Chemical Physics (Wiley, New York, 2008), pp. 267-329. URL http:// onlinelibrary.wiley.com/doi/10.1002/9780470259474.ch5/summary

14. A.F. Ordonez, O. Smirnova, Phys. Rev. A 98(6), 063428 (2018). https://doi.org/10.1103/ PhysRevA.98.063428. URL https://link.aps.org/doi/10.1103/PhysRevA.98.063428

15. A.F. Ordonez, O. Smirnova, Phys. Rev. A 99(4), 043416 (2019). https://doi.org/10.1103/ PhysRevA.99.043416. URL https://link.aps.org/doi/10.1103/PhysRevA.99.043416

16. A.F. Ordonez, O. Smirnova, Phys. Rev. A 99(4), 043417 (2019). https://doi.org/10.1103/ PhysRevA.99.043417. URL https://link.aps.org/doi/10.1103/PhysRevA.99.043417

17. L.D. Barron, J. Am. Chem. Soc. 101(1), 269 (1979). https://doi.org/10.1021/ja00495a071. URL http://dx.doi.org/10.1021/ja00495a071

18. J.A. Giordmaine, Phys. Rev. 138(6A), A1599 (1965). https://doi.org/10.1103/PhysRev.138. A1599. URL https://link.aps.org/doi/10.1103/PhysRev.138.A1599

19. P.M. Rentzepis, J.A. Giordmaine, K.W. Wecht, Phys. Rev. Lett. 16(18), 792 (1966). https:// doi.org/10.1103/PhysRevLett.16.792. URL https://link.aps.org/doi/10.1103/PhysRevLett.16. 792

20. D. Patterson, M. Schnell, J.M. Doyle, Nature 497(7450), 475 (2013). https://doi.org/10.1038/ nature12150. URL http://www.nature.com/nature/journal/v497/n7450/full/nature12150.html

21. D. Patterson, J.M. Doyle, Phys. Rev. Lett. 111(2), 023008 (2013). https://doi.org/10.1103/ PhysRevLett.111.023008. URL http://link.aps.org/doi/10.1103/PhysRevLett.111.023008

22. A. Yachmenev, S.N. Yurchenko, Phys. Rev. Lett. 117(3), 033001 (2016). https://doi.org/ 10.1103/PhysRevLett.117.033001. URL https://link.aps.org/doi/10.1103/PhysRevLett.117. 033001

23. S. Beaulieu, A. Comby, D. Descamps, B. Fabre, G.A. Garcia, R. Géneaux, A.G. Harvey, F. Légaré, Z. Mašín, L. Nahon, A.F. Ordonez, S. Petit, B. Pons, Y. Mairesse, O. Smirnova, V. Blanchet, Nat. Phys. 14(5), 484 (2018). https://doi.org/10.1038/s41567-017-0038-z. URL https://www.nature.com/articles/s41567-017-0038-z

24. N.I. Koroteev, J. Experim. Theor. Phys. 79(5), 681 (1994). URL http://jetp.ac.ru/cgi-bin/e/ index/e/79/5/p681?a=list

25. S. Woźniak, G. Wagnière, Opt. Commun. 114(1), 131 (1995). https://doi.org/10.1016/00304018(94)00498-J. URL http://www.sciencedirect.com/science/article/pii/003040189400498J

26. R. Zawodny, S. Woźniak, G. Wagnière, Opt. Commun. 130(1), 163 (1996). https://doi. org/10.1016/0030-4018(96)00224-6. URL http://www.sciencedirect.com/science/article/pii/ 0030401896002246

27. B.S. Wozniak, Mol. Phys. 90(6), 917 (1997). https://doi.org/10.1080/002689797171913. URL https://www.tandfonline.com/doi/abs/10.1080/002689797171913. Publisher: Taylor \& Francis

28. P. Fischer, A.C. Albrecht, Laser Phys. 12(8), 1177 (2002)

29. E. Gershnabel, I.S. Averbukh, Phys. Rev. Lett. 120 (8), 083204 (2018). https://doi.org/10.1103/ PhysRevLett.120.083204. URL https://link.aps.org/doi/10.1103/PhysRevLett.120.083204

30. I. Tutunnikov, E. Gershnabel, S. Gold, I.S. Averbukh, J. Phys. Chem. Lett. 9(5), 1105 (2018). https://doi.org/10.1021/acs.jpclett.7b03416. URL https://doi.org/10.1021/acs.jpclett.7b03416

31. I. Tutunnikov, J. Floß, E. Gershnabel, P. Brumer, I.S. Averbukh, Phys. Rev. A 100(4), 043406 (2019). https://doi.org/10.1103/PhysRevA.100.043406. URL https://link.aps.org/doi/ 10.1103/PhysRevA.100.043406 
32. A.A. Milner, J.A.M. Fordyce, I. MacPhail-Bartley, W. Wasserman, V. Milner, I. Tutunnikov, I.S. Averbukh, Phys. Rev. Lett. 122(22), 223201 (2019). https://doi.org/10.1103/PhysRevLett. 122.223201. URL https://link.aps.org/doi/10.1103/PhysRevLett.122.223201

33. P.V. Demekhin, A.N. Artemyev, A. Kastner, T. Baumert, Phys. Rev. Lett. 121(25), 253201 (2018). https://doi.org/10.1103/PhysRevLett.121.253201. URL https://link.aps.org/doi/10. 1103/PhysRevLett.121.253201

34. P.V. Demekhin, Phys. Rev. A 99(6), 063406 (2019). https://doi.org/10.1103/PhysRevA.99. 063406. URL https://link.aps.org/doi/10.1103/PhysRevA.99.063406

35. S. Rozen, A. Comby, E. Bloch, S. Beauvarlet, D. Descamps, B. Fabre, S. Petit, V. Blanchet, B. Pons, N. Dudovich, Y. Mairesse, Phys. Rev. X 9(3), 031004 (2019). https://doi.org/10.1103/ PhysRevX.9.031004. URL https://link.aps.org/doi/10.1103/PhysRevX.9.031004

36. A.F. Ordonez, O. Smirnova, [physics] (2020). URL http://arxiv.org/abs/2009.03660

37. A.F. Ordonez, O. Smirnova, [physics] (2020). URL http://arxiv.org/abs/2009.03655

38. D.L. Andrews, T. Thirunamachandran, J. Chem. Phys. 67(11), 5026 (1977). https://doi.org/10. 1063/1.434725. URL http://aip.scitation.org/doi/citedby/10.1063/1.434725

39. D.J. Cook, R.M. Hochstrasser, Opt. Lett. 25(16), 1210 (2000). https://doi.org/10.1364/OL.25. 001210. URL https://www.osapublishing.org/ol/abstract.cfm?uri=ol-25-16-1210

40. R.R. Freeman, P.H. Bucksbaum, H. Milchberg, S. Darack, D. Schumacher, M.E. Geusic, Phys. Rev. Lett. 59(10), 1092 (1987). https://doi.org/10.1103/PhysRevLett.59.1092. URL https:// link.aps.org/doi/10.1103/PhysRevLett.59.1092

Open Access This chapter is licensed under the terms of the Creative Commons Attribution 4.0 International License (http://creativecommons.org/licenses/by/4.0/), which permits use, sharing, adaptation, distribution and reproduction in any medium or format, as long as you give appropriate credit to the original author(s) and the source, provide a link to the Creative Commons license and indicate if changes were made.

The images or other third party material in this chapter are included in the chapter's Creative Commons license, unless indicated otherwise in a credit line to the material. If material is not included in the chapter's Creative Commons license and your intended use is not permitted by statutory regulation or exceeds the permitted use, you will need to obtain permission directly from the copyright holder. 